\title{
Breath testing as a method for detecting lung cancer
}

\section{Immanuels Taivans, Maris Bukovskis, Gunta Strazda \& Normunds Jurka}

To cite this article: Immanuels Taivans, Maris Bukovskis, Gunta Strazda \& Normunds Jurka (2014) Breath testing as a method for detecting lung cancer, Expert Review of Anticancer Therapy, 14:2, 121-123

To link to this article: $\underline{\text { http://dx.doi.org/10.1586/14737140.2013.866044 }}$

\section{曲 Published online: 28 Jan 2014.}

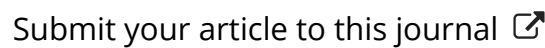

Џlll Article views: 430

Q View related articles $₫$

View Crossmark data $\asymp$ 


\section{Breath testing as a method for detecting lung cancer}

Expert Rev. Anticancer Ther. 14(2), 121-123 (2014)
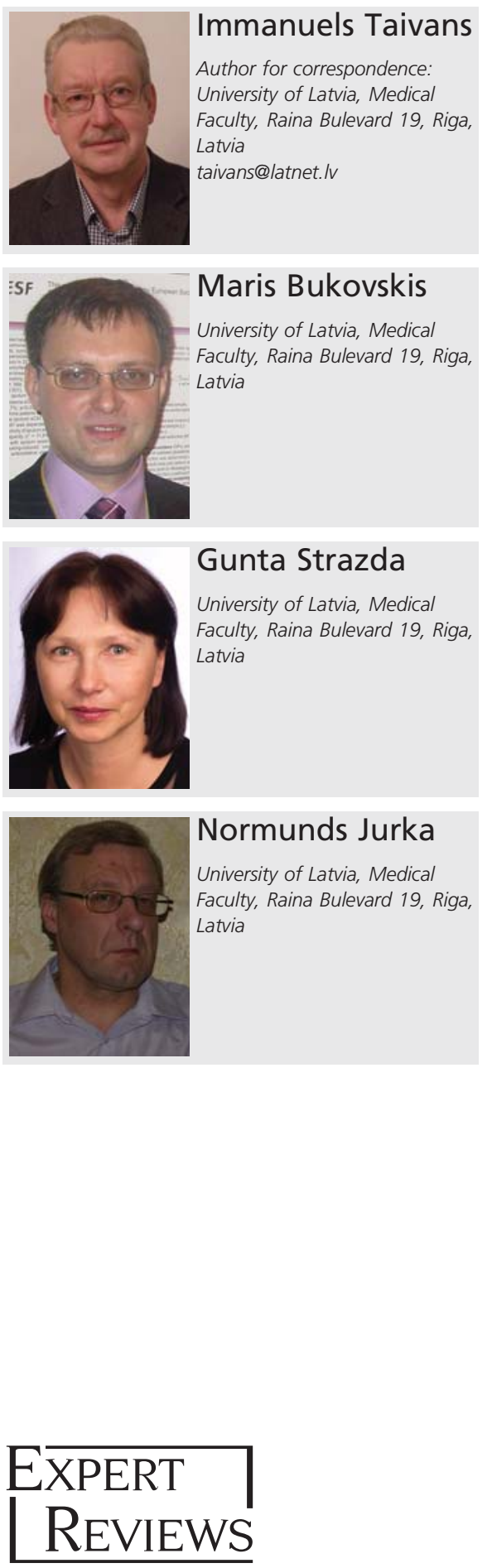

Early diagnosis of lung cancer is important due to high mortality in late stages of the disease. An ideal approach for population screening could be the breath analysis, due to its non-invasiveness, simplicity and cheapness. Using sensitive methods of analysis like gas chromatography/mass spectrometry in exhaled air of cancer patients were discovered some volatile organic compounds - possible candidates for cancer markers. However, these compounds were not specific for cancer cells. At the same time, integrative approaches used to analyze the exhaled breath have demonstrated high sensitivity and specificity of this method for lung cancer diagnosis. Such integrative approaches include detection of smell prints by electronic nose or integrated analysis of wide range of volatile organic compounds detected by gas chromatography/mass spectrometry or related methods. Modern statistical pattern recognition systems like logistic regression analysis, support vector machine or analysis by artificial neuronal network may improve diagnostic accuracy.

Lung cancer is the most common cancer worldwide. The diagnosis of early stage lung cancer is essential and substantially determines the life expectancy. Five-year survival is expected in $58-73 \%$ of patients with stage I lung cancer. For later stages of disease the 5-year survival rate is only $3.5 \%$. Unfortunately, only $15 \%$ of lung cancer cases are diagnosed at an early stage. Over half of people with lung cancer die within the first year of being diagnosed [1].

The problem is that available diagnostic methods, that is, sputum cytological analysis and chest $\mathrm{x}$-ray, are not sensitive enough, and they are expensive or invasive. They do not fit well for population screening [2,3]. An ideal approach could be breath analysis due to its non-invasiveness, simplicity and cheapness. It is especially suited for lung cancer screening and monitoring as other methods like computerized tomography are expensive and cannot be applied as frequently as necessary due to irradiation.

Integrative approaches used to analyze the exhaled breath have demonstrated high sensitivity and specificity of this method for lung cancer diagnosis. Such integrative approaches include detection of breathprint by electronic nose or integrated analysis of wide range of volatile organic compounds (VOCs) detected by gas chromatography/mass spectrometry or related methods [4-6].

At the same time, attempts to identify particular compounds specific for lung cancer have not been successful up to present time. Several laboratories have tried to select specific cancer markers from the headspace of cancer cell cultures. Some of the compounds selected were alcanes or methylated alcanes like decane, nonadecane, eicosane [7], 2-methylpentane, spirits like 2-ethyl1-hexanol and ketones like 2-pentadecanone $[8]$. One problem with headspace measurements of cell cultures is that the cells take up the chemical compounds from the medium, metabolize them and then release the products of the metabolism. Thus, the product of cells is dependent on the environment in which cells are located. Besides, different tumor cell types in the same medium metabolize different compounds and release distinct 
VOCs [9]. And finally, these data do not prove that tumor cells are the exclusive source of mentioned putative cancer markers.

Another approach is to select specific VOCs from the exhaled air of lung cancer patients. Relevant studies have shown that some compounds in the breath of cancer patients are present in elevated, but some in diminished concentrations, when compared with healthy controls. However, no specific compounds characteristic exclusively for cancer cells have ever been found. In these studies, the question remains, what is the source of VOCs in the exhaled air? Is the amount of VOCs released from the tumor cells in the exhaled air sufficient to meet the detection limit of the method used? Previous studies have examined the exhaled air composed of dead space and alveolar fractions. VOCs originating from lung tumors are expected to be present in dead space fraction. Alveolar fraction is expected to contain VOCs from blood circulation, released by different body cells, including the tumor cells of the lung. In mixed exhaled air, the content of VOCs released by lung tumor cells may be low and may not exceed the detection limit. At the same time, the alveolar air may contain VOCs released by tumors from any location.

Apart from VOCs, tumor cells produce wide range of cytokines like IL-4, IL- 6 , IL-11, IL-15, TNF- $\alpha$, TGF- $\beta$ and others, which activate body's immune system and change the metabolism of wide range of body cells [10,11]. An example of cancer influence on the body is cachexia, which is characterized by hypermetabolism and generalized muscle wasting. Recently, a cytokine responsible for these changes was discovered - it is a TNF-like weak inducer of apoptosis. TNF-like weak inducer of apoptosis activates various proteolytic pathways and stimulates the degradation of myofibril proteins [12].

Interesting is the observation of Poli et al. [13], who measured a set of VOCs in exhaled air before and 4 weeks after the tumor resection. VOCs present in elevated concentrations before the surgery remained at the same level after the resection. The only exception was isoprene, the concentration levels of which dropped to the level of healthy controls. Re-examination of the same subjects 3 years later showed that only after this time period such VOCs as pentane, benzene, ethylbenzene and toluene have returned to the levels observed in controls [14]. Authors concluded "that exhaled VOCs do not, or only in part, derive from cancerogenous tissue, being their concentration is unaffected by surgical removal ... A possible explanation of these data is that exhaled VOCs cannot be considered biomarkers of lung cancer per se, rather epiphenomenon which accompany lung cancer development...” [14].

However, the pathogenesis of this epiphenomenon is still unclear. Persistence of elevated levels of VOCs in exhaled air even 1 month after the removal of tumor cannot be explained by substances produced by the tumor itself. Evidently during the process of cancerogenesis some longstanding changes develop also outside the tumor. These changes may be of immunological or genetic origin. Based on observations that VOCs pattern did not differ between the tumor stages, Phillips et al. postulated the mechanism by which polymorphic isoforms of cytochrome P450 family enzymes are formed in the early stages of cancerogenesis. These enzymes oxidize DNA to induce cancerogenic mutations and oxidize membrane phospholipids with production of alcanes and methylated alcanes, which are exhaled with breath [15].

Whatever is the intimate mechanism of VOCs production, it is clear that in cancerous state their concentrations in exhaled air become changed in a typical manner, which may be uncovered by large-scale analysis.

Applicable for such a purpose is electronic nose. Diagnostics using this device is simple, sufficiently accurate, inexpensive, non-invasive, allows online diagnosis and can differentiate heterogeneous disorders. The information provided by this technique is not based on detecting single and separate molecular signals, but is exclusively derived from pattern recognition among an array of signals by using powerful bioinformatics [16].

Electronic nose is an instrument made up of different kind of chemical sensors combined with a pattern recognition system. The measurement in electronic nose is based on the different mechanisms - electrical resistance, ion-gas or colorimetric sensor response that differs regarding VOCs molecular pattern [17]. If there has been no change in the composition of the air, the resistance of sensors does not change. When sensors are exposed to VOCs, they absorb the analyte and subsequently change their electric resistance. Each sensor may give response to different VOCs. At the same time, several sensors may react with the same VOC. A unique response curve combination is recorded, containing the information to allow discrimination of the different samples. If we examine patient groups with different diseases, pattern recognition system develops typical average 'breathprint' characteristic for patients with similar diagnosis [16].

Clinical studies have shown potential of exhaled breath analysis by electronic nose. Machado et al. in their study found that exhaled air analysis demonstrated discrimination between samples from patients with lung cancer and those from other groups. In the validation study, the electronic nose had $71.4 \%$ sensitivity and $91.9 \%$ specificity for detection of lung cancer. Positive and negative predictive values were 66.6 and $93.4 \%$, respectively [18].

Dragonieri et al. in two studies demonstrated that VOC patterns of exhaled breath discriminates patients with lung cancer from chronic obstructive lung disease (COPD) patients, as well as healthy controls and malignant pleural mesothelioma from subjects with similar occupational asbestos exposure without malignant pleural mesothelioma, and from healthy controls. In all three studies, the conclusion was that the electronic nose may qualify as a non-invasive diagnostic or screening tool for lung malignancy in the future $[19,20]$.

In other study, the breath of volunteers was examined by a tailor-made array of cross-reactive nanosensors based on organically functionalized gold nanoparticles and gas chromatography linked to the mass spectrometry. The results showed that the gold nanosensor array could differentiate between 'healthy' and 'cancerous' breath, and, furthermore, between the breath of 
patients having different cancer types - lung, breast, colorectal or prostate cancer. The gas chromatography/mass spectrometry results showed that each cancer could have a unique pattern of VOCs, when compared with healthy states, but not when compared with other cancer types [21].

A significant challenge to exhaled breath analysis by electronic nose is posed by the heterogeneity of patients. Both lung cancer and COPD are diseases associated with cigarette smoking, and both coexist in many patients. In many patients, advanced lung cancer is comorbid with complications like post-obstructive atelectasis, pneumonia, exudative pleuritis or carcinomatous lymphanghoitis, which can significantly change the VOCs pattern in exhaled breath. Finally, patient smoking status, smoking history and even diet may confound prognostic results of analysis.

Evidence that discrimination is possible comes from studies trying to differentiate patients with combined diseases, for example, lung cancer and COPD. Two hundred and eleven out of 221 (95.5\%) lung cancer patients were classified correctly in a study population consisting of patients with lung cancer alone, COPD alone, lung cancer with concomitant
COPD and healthy volunteers. The main classification error occurred between cancer and cancer COPD group, which is not clinically significant [22].

Development of specific mathematical models for diagnosis of lung cancer using exhaled breath by electronic nose may improve diagnostic accuracy. In all studies mentioned above, the principal component or canonical discriminant analyses were used for predictive purposes. Modern statistical pattern recognition systems offer an opportunity to choose methods with potentially best predictive value, for example, logistic regression analysis, support vector machine or even artificial neuronal network approach [23].

\section{Financial \& competing interests disclosure}

The authors have no relevant affiliations or financial involvement with any organization or entity with a financial interest in or financial conflict with the subject matter or materials discussed in the manuscript. This includes employment, consultancies, honoraria, stock ownership or options, expert testimony, grants or patents received or pending or royalties.

No writing assistance was utilized in the production of this manuscript.

\section{References}

1 American Cancer Society. Cancer Facts \& Figures 2012. American Cancer Society, Atlanta, USA (2012).

2 Sing A, Freudenberg N, Kortsik C et al. Comparison of the sensitivity of sputum and brush cytology in the diagnosis of lung carcinomas. Acta Cytol. 41(2), 399-408 (1997).

3 Frost JK, Ball WC Jr, Levin ML et al. Early lung cancer detection: results of the initial (prevalence) radiologic and cytologic screening in the Johns Hopkins study. Am. Rev. Respir. Dis. 130, 549-554 (1984).

4 Van de Kant KDG, van der Sande LJTM, Jöbsis Q et al. Clinical use of exhaled volatile organic compounds in pulmonary diseases: a systematic review. Respir. Res. 13, 117 (2012).

5 Horvath I, Lazar Z, Giulai N et al. Exhaled biomarkers in lung cancer. Eur. Respir. J. 34, 261-275 (2009).

6 Van Berkel JJBN, Dallinga JW, Möller GM et al. Development of accurate classification method based on the analysis of volatile organic compounds from human exhaled air. J. Chromatogr. B Analyt. Technol. Biomed. Life Sci. 861, 101-107 (2008).

7 Hu YJ, Qiu YH, Chen EG et al. Determination of volatile organic compounds in lung cancer cell lines and lung tissue. Zhejiang Da Xue Bao Yi Xue Ban 39(3), 278-84 (2010).

8 Sponring A, Filipac W, Mikowini T et al. Release of volatile organic compounds from the lung cancer cell line NCI-H2087 in vitro. Anticancer Res. 29, 419-426 (2009).
9 Mochalski P, Sponring A, King J et al. Release and uptake of volatile organic compounds by human hepatocellular carcinoma cells (HepG2) in vitro. Cancer Cell Int. 13, 72 (2013).

10 Haselbag S, Fleuren GJ, Baelde JJ et al. Cytokine profile of cervical cancer cells, Gynocol. Oncol. 83(2), 235-243 (2001).

11 Yamamoto T, Kimura T, Ueta E et al. Characteristic cytokine patterns in cancer cells and infiltrating lymphocytes in oral squamous cell carcinomas and the influence of chemoradiation combined with immunotherapy on these patterns. Oncology 64(4), 407-415 (2003).

12 Bhatanagar S, Kumar A. The TWEAKFn14 system: breaking the silence of cytokine-induced skeletal muscle wasting. Curr. Mol. Med. 12(1), 3-13 (2012).

13 Poli D, Carbogniani P, Corradi M et al. Exhaled volatile organic compound in patients with non-small cell lung cancer: cross sectional and nested short-term followup study. Respir. Res. 6, 71 (2005).

14 Poli D, Goldoni M, Caglieri A. Breath analysis in non-small cell lung cancer patients after surgical tumour resection. Acta. Biomed. 79(Suppl. 1), 64-72 (2008).

15 Phillips M, Cataneo RN, Cummin ARC et al. Detection of lung cancer with volatile markers in the breath. Chest 123(6), 2115 (2003).

16 Gardner JW, Bartlett PN. Electronic noses. Principles and applications. Meas. Sci. Technol. 11, 1087 (2000).

17 Mazzone PJ, Wang XF, Xu Y. Exhaled breath analysis with a colorimetric sensor array for the identification and characterization of lung cancer. J. Thorac. Oncol. 7(1), 137-142 (2012).

18 Machado RF, Laskowski D, Deffenderfer O et al. Detection of lung cancer by sensor array analyses of exhaled breath. Am. J. Respir. Crit. Care Med. 171, 1286-1291 (2005).

19 Dragonieri S, Annemaa JT, Schot R et al. An electronic nose in the discrimination of patients with non-small cell lung cancer and COPD. Lung Cancer 64, 166-170 (2009).

20 Dragonieri S, van der Scheeb MP, Massaroc $\mathrm{T}$ et al. An electronic nose distinguishes exhaled breath of patients with malignant pleural mesothelioma from controls. Lung Cancer 75, 326-331 (2012).

21 Peng G, Hakim M, Broza YY et al. Detection of lung, breast, colorectal, and prostate cancers from exhaled breath using a single array of nanosensors. Br. J. Cancer 103, 542-551 (2010).

22 Strazda G, Bukovskis M, Kopeika U et al. Analysis of exhaled breath with electronic nose and discrimination of lung cancer and COPD by logistic regression analysis. European Respiratory Society. Barcelona, Spain, 7-11 September 2013 (Poster 2891).

23 Basanta M, Ibrahim B, Dockry R et al. Exhaled volatile organic compounds for phenotyping chronic obstructive pulmonary disease: a cross-sectional study. Respir. Res. 23(13), 72 (2012). 